\title{
Project report: new generation intelligent building platform techniques
}

\author{
Qianchuan Zhao ${ }^{1 *}$, Li Xia $^{1}$ (1) and Ziyan Jiang ${ }^{2}$
}

\author{
* Correspondence: \\ zhaoqc@tsinghua.edu.cn \\ ${ }^{1}$ Center for Intelligent and Networked \\ Systems (CFINS), Department of \\ Automation and TNList, Tsinghua \\ University, Beijing 100084, China \\ Full list of author information is \\ available at the end of the article
}

\begin{abstract}
Background: Governments and societies all over the world pay more and more attention to the research and development of smart buildings. There are numerous research projects on smart buildings that have been or being conducted worldwide.

Case presentation: In this project report, we give an introduction about a newly launched research project sponsored by the Chinese central government. This project aims to combine information technology and control \& optimization methodology to address challenging problems in building energy. To study and implement a decentralized control and optimization paradigm in smart buildings is one of the key tasks of this project.

Conclusions: The combination of informatics and energy is a very important viewpoint to study the management and operation of modern buildings. Research activities in this area shall contribute a lot to the invention and development of new building industrial opportunities.
\end{abstract}

Keywords: Smart building, Building energy, Cyber physical system, Decentralized control

\section{Background}

As one of the most important parts of infrastructure supporting daily life of human society, buildings' efficient and safe operation is a critical issue. Just taking building energy consumption as an example, buildings contribute a significant part of the total energy consumption in many developed and developing countries. For example, in 2016, buildings (residential and commercial sectors) consume about $40 \%$ of the total energy and $70 \%$ of the electricity in the United States, according to the annual energy review of the U.S. Energy Information Administration (https://www.eia.gov/tools/faqs/ faq.php?id=86\&t=1). The numbers in $\mathrm{EU}$ and other developed countries are also similar (https://ec.europa.eu/energy/en/topics/energy-efficiency/buildings) (Berardi 2015). In China, the energy consumption in buildings has risen from $10 \%$ to $25 \%$ of the overall energy consumption of the country since 1978, and is predicted to be increased to $35 \%$ in the coming years (Jia et al. 2014).

However, the efficient and safe operation control and management of buildings is extremely challenging due to the strong dependence of building performances on continuously varying functionalities of buildings, occupant behaviors, outdoor environments, diversified structure and building materials, customized operation management

(c) The Author(s). 2018 Open Access This article is distributed under the terms of the Creative Commons Attribution 4.0 International License (http://creativecommons.org/licenses/by/4.0/), which permits unrestricted use, distribution, and reproduction in any medium, provided you give appropriate credit to the original author(s) and the source, provide a link to the Creative Commons license, and indicate if changes were made. 
strategies, and so on. Thus, the intelligent building control and management is by nature a cross-disciplinary research, and requires one to use the knowledge (such as models on building dynamics, occupant behaviors, and outdoor environments) and the methodology (such as optimization and online decision-making theory) from multiple fields.

In recent decades, the fast development of information technology has significantly changed the engineering, economical, and social fields of human society. The research on smart buildings also thrives, although most of our current buildings are still working in an out-of-date manner. Utilizing modern information and control technology (ICT) to rebuild the information infrastructure of buildings has fundamental effects on the research of smart buildings. Since building is a very important part of the whole energy system in human world, the combination of informatics and energy plays a significant role in the research of efficient management and operation of modern buildings. The research of smart buildings in China is developing fast. However, the deployment of building management systems (BMS) in China still needs numerous efforts to make it become a standard. The current BMS is in a centralized form and it requires a lot of manpower to make configurations during the deployment. The scalability and robustness are also potential concerns. How to treat these challenges is an interesting research topic.

Governments and societies all over the world pay more and more attention to the research and development of smart buildings. There are numerous research projects on smart buildings that have been or being conducted worldwide. For example, the Information Society of European Commission has sponsored a series of projects (35 projects listed on the official website of EU, see (http://ec.europa.eu/information_society/activities/sustainable_growth/ funding/prj_buidings/index_en.htm) for more information) to support the sustainable growth of Europe. These projects aim at applying ICT to buildings for energy saving and intelligent management. The NSF of the U.S. also launched a series of research projects in smart buildings, e.g., a \$2-million grant was funded to scholars of MIT and Boston University to explore substantial carbon footprint and energy cost reductions in buildings (https:// www.bu.edu/sustainability/nsf-greenlights-sustainable-building-project/). Compared to these

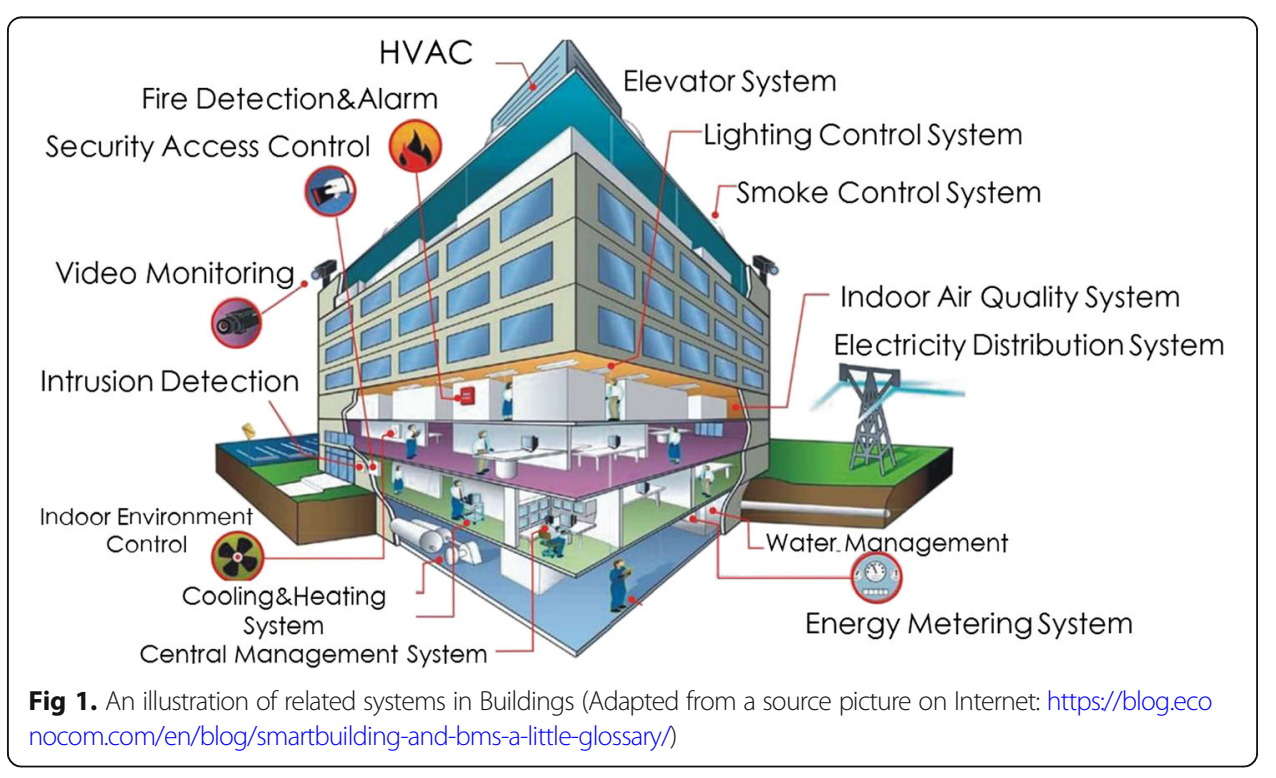


projects, the main feature of our project is the decentralized framework, which is a beneficial exploration of self-organization, scalability, and robustness of ICT in smart buildings (Fig. 1).

\section{Case presentation}

\section{Project description}

Recently, China launched a National Key Research and Development Program entitled new generation intelligent building platform techniques (2017.07-2020.12) with Grant No. 2017YFC0704100. Such category of programs is the top tier of scientific projects sponsored by the Chinese government, which reflects the prior research direction national wide. A typical financial amount of such project is around 10 million US dollars and it will involve many partners diverse from universities, research institutes, industrial companies, etc.

The aim of this project (Grant No. 2017YFC0704100) is to introduce advanced information and control technologies including system modeling, decentralized control and optimization, Internet of Things (IoT) to address the challenges arising from the intelligent operation of building devices in a highly dynamical environment. The paradigm of decentralized control and optimization is the key feature of this new building platform. In the decentralized framework of smart buildings, there does not exist a centralized node or computer to coordinate the whole control system. All the nodes in the building are at a peer level and they are self-organized together to accomplish various functions of the building. This decentralized framework of ICT can alleviate the deficiencies caused by the traditional centralized control schemes, such as the difficulties of automatic configuration and deployment, self-organization, scalability, robustness, etc. As illustrated in Fig. 2, the new framework of the decentralized operating system is composed of many building space units, which are working in a self-organized way. Each unit in the topology of the right side of Fig. 2 includes many intelligent building devices, such as sensors and actuators of buildings. More importantly, each space unit has a computing node, which monitors and controls all the devices inside this space unit. Computing nodes also communicate with its neighbors in a peer-to-peer way, which makes the total operating system work in a decentralized style.

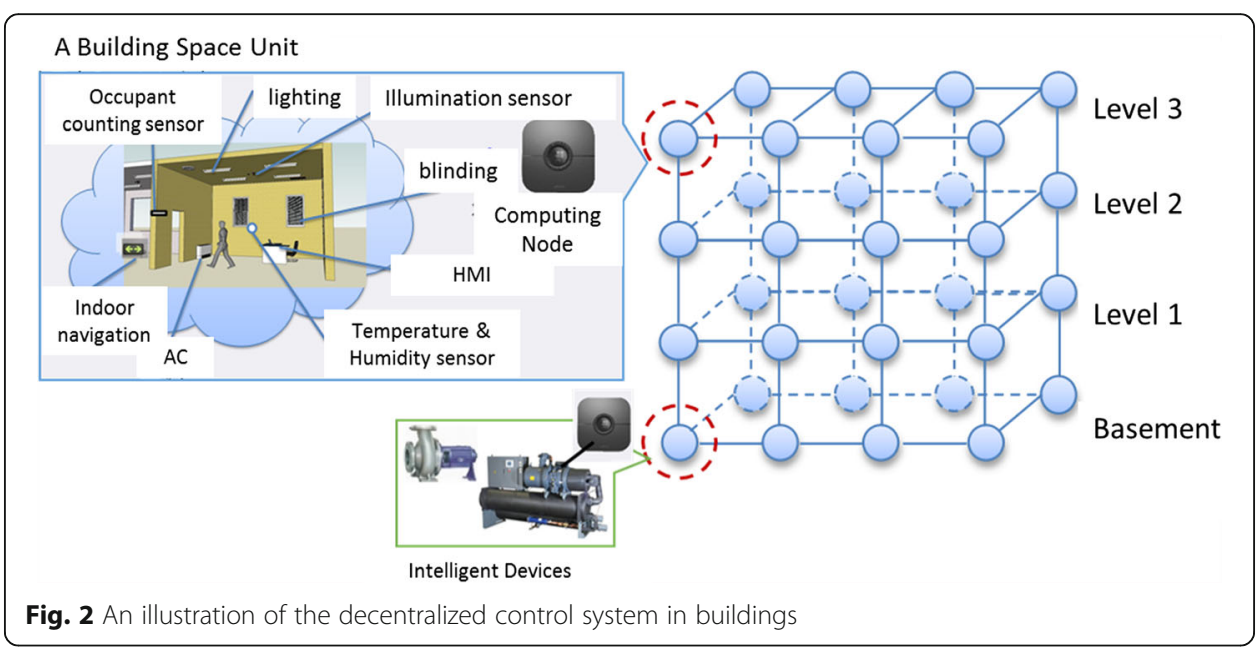


Some deliveries of this project include a decentralized operating system for smart buildings and intelligent electromechanical devices working in decentralized form. The system will be deployed in at least 5 demonstration buildings, more than 100,000 square meters. These buildings are commercial complexes and located in different climate zones of China, two in South China, one in East China, and two in North China. The operating system should accommodate more than 5000 intelligent nodes, 30 types of electromechanical devices, and 20 software applications. The accumulative localization errors of indoor occupants should be less than $10 \%$.

\section{Project partners}

Prof. Qianchuan Zhao from the Center for Intelligent and Networked Systems (CFINS), Department of Automation, Tsinghua University is the PI of the project. Prof. Jili Zhang and Prof. Guowei Wu from Dalian University of Technology, Prof. Jianchun Xing and Prof. Qiliang Yang from PLA University of Science and Technology, Prof. Qiansheng Fang and Prof. Zhenya Zhang from Anhui Jianzhu University, and Dr. Ziyan Jiang from Tsinghua University are Co-PIs. The team also includes researchers and engineers from China Academy of Building Research, China Institute of Building Standard Design \& Research, China Architecture Design Group and other industrial partners. The industrial partners have three types of companies. One type is the real estate companies, such as China Resources (Holdings), which provide demonstration buildings to deploy our technology developed in this project. The second type is the manufacturers of building devices, such as Gree Electric Appliances and Sinro Air-Conditioning Company, which provide the manufacturing of intelligent building devices. The third type is the system integrators, such as Tsinghua Tongfang, which deploy the whole system to demonstration buildings.

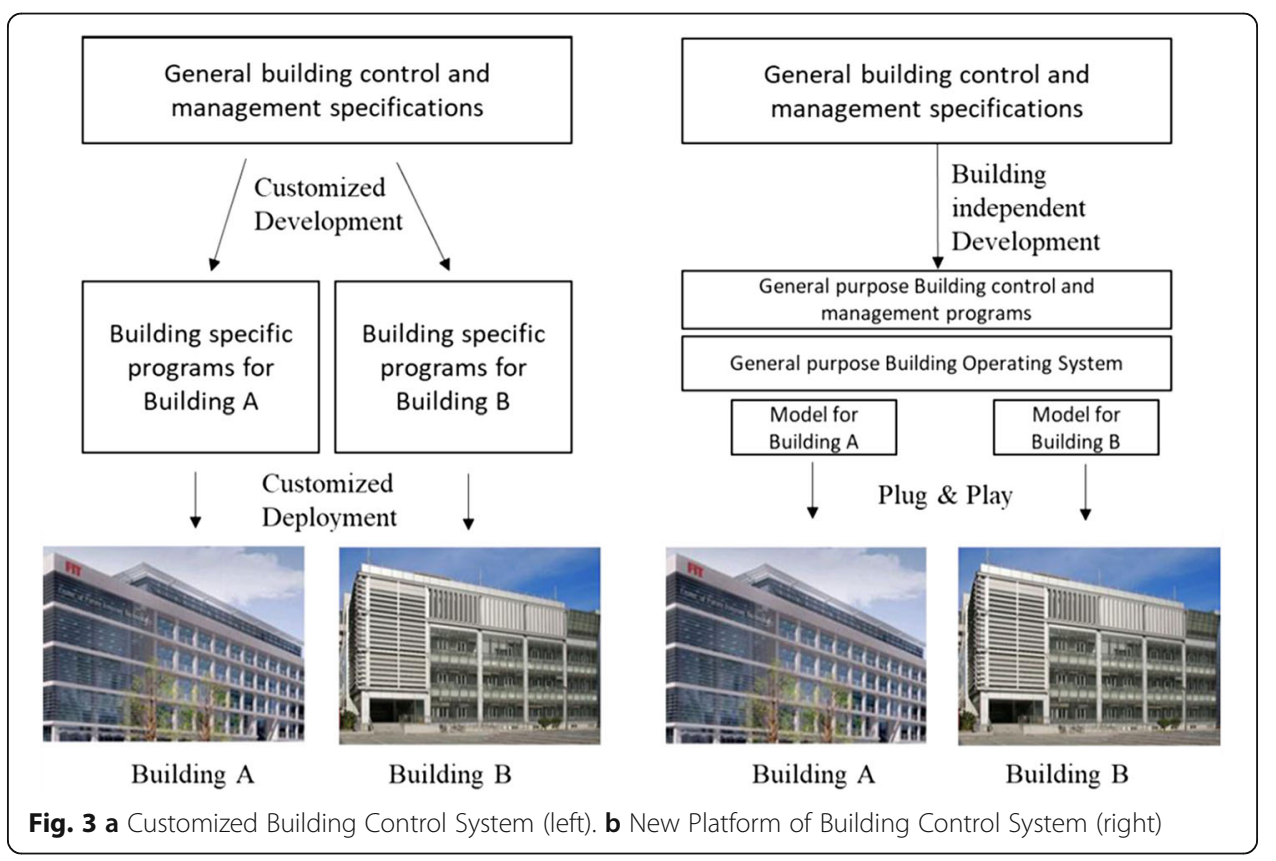




\section{Discussion and evaluation}

The team employs cyber-physical systems (CPS) as the overall modeling framework. The advantage of this strategy is that the physics underlying building dynamics can be explicitly considered. A new architecture of building control and management systems will be developed to reduce design, deployment, and maintenance cost. Different from traditional way of developing building control systems as shown in Fig. 3a, by considering physics side of buildings, general purpose building control programs will be developed on top of a general purpose building operating system, as shown in Fig. 3b. The building operating system needs to hide the diversified structures of individual buildings and present interfaces to the general purpose building control and management programs (application programs) to access the abstract model of buildings. The application programs are developed based on standard building models instead of specific buildings.

\section{Conclusions}

Although China is still a developing country, China has a fast investment rate of infrastructure construction all over the world. For the sector of building industry, China has more than $50 \%$ of the constructed super high-rise buildings in the world (https://en.wi kipedia.org/wiki/List_of_tallest_buildings) and more are under construction. The combination of informatics and energy is a very important viewpoint to study the management and operation of modern buildings. The Chinese government has realized the importance of buildings in the whole energy system and research \& development. A lot of research grants and programs are being announced, according to the national 5 -years research plan of China. These emerging research activities also contribute a lot to the invention and development of new industrial opportunities.

\section{Acknowledgements}

The authors would like to present their appreciation to the entire research team of this project, who have contributed and been contributing to advance the progress of this project.

Funding

This work was supported by the National Key Research and Development Program of China with Grant No. 2017 FC0704100.

Availability of data and materials

This report does not include specific experiment data or materials.

Authors' contributions

QZ and LX mainly composed the manuscript and ZJ provided the details about the decentralized testbed. All authors read and approved the final manuscript.

Competing interests

The authors declare that they have no competing interests.

\section{Publisher's Note}

Springer Nature remains neutral with regard to jurisdictional claims in published maps and institutional affiliations.

Author details

${ }^{1}$ Center for Intelligent and Networked Systems (CFINS), Department of Automation and TNList, Tsinghua University, Beijing 100084, China. ${ }^{2}$ Building Energy Research Center, Tsinghua University, Beijing 100084, China.

Received: 17 January 2018 Accepted: 16 March 2018

Published online: 26 July 2018

\section{References}

Berardi U (2015) Building energy consumption in US, EU, and BRIC countries. Procedia Engineering 118:128-136.

Jia QS, Zhao Q, Darabi H, Huang HS, Becerik-Gerber B, Sandberg H, Johansson KH (2014) Smart Building Technology. IEEE Robot Autom Mag 21(2):18-20. 